\title{
PCA-based artifact removal algorithm for stroke detection using UWB radar imaging
}

\author{
Elisa Ricci $^{1} \cdot$ Simone di Domenico $^{1} \cdot$ Ernestina Cianca $^{1}\left[{ }^{1} \cdot\right.$ Tommaso Rossi $^{1}$ \\ Marina Diomedi ${ }^{2}$
}

Received: 16 February 2016 / Accepted: 2 September 2016

(C) International Federation for Medical and Biological Engineering 2016

\begin{abstract}
Stroke patients should be dispatched at the highest level of care available in the shortest time. In this context, a transportable system in specialized ambulances, able to evaluate the presence of an acute brain lesion in a short time interval (i.e., few minutes), could shorten delay of treatment. UWB radar imaging is an emerging diagnostic branch that has great potential for the implementation of a transportable and low-cost device. Transportability, low cost and short response time pose challenges to the signal processing algorithms of the backscattered signals as they should guarantee good performance with a reasonably low number of antennas and low computational complexity, tightly related to the response time of the device. The paper shows that a PCA-based preprocessing algorithm can: (1) achieve good performance already with a computationally simple beamforming algorithm; (2) outperform stateof-the-art preprocessing algorithms; (3) enable a further improvement in the performance (and/or decrease in the number of antennas) by using a multistatic approach with
\end{abstract}

Ernestina Cianca

cianca@ing.uniroma2.it

Elisa Ricci

elisa.ricci.ing@gmail.com

Simone di Domenico

simone.di.domenico@uniroma2.it

Tommaso Rossi

tommaso.rossi@uniroma2.it

Marina Diomedi

maria.diomedi@uniroma2.it

1 Department of Electronic Engineering, University of Rome "Tor Vergata", Rome, Italy

2 Neuroscience Department, Policlinic of “Tor Vergata”, Rome, Italy just a modest increase in computational complexity. This is an important result toward the implementation of such a diagnostic device that could play an important role in emergency scenario.

Keywords Brain stroke detection · Microwave UWB radar - Artifact removal $\cdot$ PCA

\section{Introduction}

According to the World Health Organization, stroke is the second cause of death above the age of 60 years and the first cause of dependence worldwide.

About $87 \%$ of all strokes are ischemic strokes [14] and are caused by an inadequate blood supply to part of the brain. Intracranial hemorrhage, caused by bleeding mainly into parenchymal brain tissue, is responsible for 9 to $27 \%$ of all strokes worldwide [5].

Given the high incidence and significant morbidity and mortality of stroke, a rapid diagnosis and treatment are very important, both in ischemic and in hemorrhagic strokes. Neuroimaging techniques play a fundamental role in acute stroke process being the first step in stroke pathogenesis evaluation. In the pre-computerized tomography era, it was impossible to reliably distinguish between cerebral hemorrhage and infarction. Nowadays, early after stroke, CT is used to identify cerebral hemorrhage with almost complete sensitivity and specificity.

As for the heart attack, stroke patients should be dispatched at the highest level of care available in the shortest time. Statewide standardization of telecommunication programs, stroke education modules and care protocols are recommended to facilitate the recognition of stroke in the acute phase, provide adequate pre-hospital stroke care by 
emergency medical services and a rapid assessment and management of patients [9]. In this context, a transportable system in specialized ambulances, able to evaluate the presence of an acute brain lesion, could shorten delay of treatment. In particular, the identification of a hemorrhagic nature of the event could help to differentiate the subgroup of "hemorrhagic" patients from "possible ischemic" patients and select the most adequate hospital destinations.

Microwave imaging is an emerging diagnostic branch that has great potential for the implementation of this transportable and low-cost device [24, 25]. MWI is based on the observation that different tissues or the same tissues but in different functional conditions (i.e., cancerous and healthy tissue) have different dielectric properties in the microwave band [2]. Two main MWI approaches exist: (1) microwave tomography; (2) UWB radar imaging.

Microwave tomography determines morphological (location, size and shape) and electromagnetic characteristics (permittivity, conductivity and magnetic permeability) of any abnormal examined tissue [7]. The output is the reconstruction of the complete dielectric profile of the crossed tissues. The tissue reconstruction requires the solution of a very complex mathematical inverse problem. Moreover, the tomography approach requires a classification process, and hence, a training phase to construct a database with many clinical cases and a large amount of a priori and ad hoc information. In UWB radar imaging, broadband pulses are emitted from antennas properly located around the object to be analyzed, non-necessarily in contact with the object. Backscattered signals are collected by the same antennas. Arrival times and amplitudes of backscattered signals are processed by a beamforming algorithm to locate the scattering points, i.e., the points in which there is a transition from one type of tissue to another. The output is a map of the backscattering energy, i.e., an image where the pixels with higher energy represent potential "abnormal" areas.

So far, some feasibility studies and proof of concepts on the use of MWI for stroke detection have been reported [6, $8,12,13,18,23]$. A relevant work has been done at Chalmers University $[6,12,18]$, where clinical trials are ongoing. That device is based on the microwave tomography approach.

Less advanced is the study of UWB radar imaging for stroke detection. As a matter of fact, an UWB radar imaging has great potential for the implementation of a simple, transportable and low-cost device, which would have the further advantage of not requiring a training phase for classification purposes [17]. Main challenges are related to:

1. Antennas design.

2. The trade-off between computational complexity, which is related to the costs and response time of the device and performance (i.e., stroke detection capability).

Several works have been done on the antennas design [13, 28]. In this paper, we focus on the second challenge. Most of the works on UWB radar for stroke detection consider a monostatic system and the delay-and-sum (DAS) algorithm $[8,13]$, which was originally proposed for breast cancer detection [11]. The DAS algorithm has the attracting feature of having low computational complexity but it offers limited robustness to artifacts. To achieve good performance using the DAS algorithm, a very high number of antennas must be considered. For instance, in [13], 16 and 32 antennas are used. To improve the performance and reduce the number of antennas, much more complex beamforming algorithms have been proposed such as the MIST, microwave imaging space-time [1, 16], also originally proposed for breast cancer detection. In [20], the MIST algorithm has been extended to stroke detection and to the multistatic case. As shown in [20], multistatic MIST can provide very good performance also with a low number of antennas (such as 8). However, this algorithm has a very high computational complexity, especially in case of a multistatic system, which might result in a response time of the order of hours. Therefore, this is not a viable solution for the stroke detection device considered in this paper.

Modifications to the DAS algorithm have been proposed in [27] for breast cancer and extended to stroke detection in [21], which are proved to improve the robustness of DAS to clutter. Also in this case, the improved performance is achieved at expense of an increased computational complexity.

In this paper, rather than on the processing algorithm, we focus on the preprocessing phase that is needed to remove artifacts. As a matter of fact, the preprocessing is performed once on the received signals while the processing algorithm is applied on each pixel. Therefore, keeping low the computational complexity of the processing algorithm and using a more powerful preprocessing algorithm represent a more effective solution to improve the trade-off performance-computational complexity.

So far, no much effort has been put on the design of an optimized artifact removal algorithm for stroke detection. In [20], the Wiener filtering, originally used for breast cancer detection, has been optimized for stroke detection and extended to the multistatic case. Moreover, a novel algorithm based on PLSR (partial least square regression) has been proposed and compared with the Wiener filtering. In [15], two novel algorithms for stroke detection have been proposed and their performance assessed. They are based on the symmetry with respect to the head midline that divides the head in left and right hemisphere. 
Fig. 1 Overall system block diagram

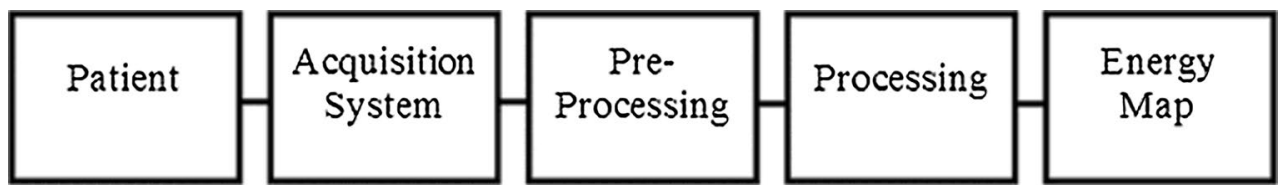

This paper proposes a low complexity preprocessing algorithm based on principal component analysis (PCA) processing. PCA has been used for artifact removal in land mines and through the wall UWB radar applications [10, 26], but it has not been considered and optimized for stroke detection. For breast cancer detection, the singular value decomposition (SVD) has been used in [4] to separate the artifacts and lesion subspaces and then remove the artifacts. However, as it will be shown in this paper, the algorithm considered in [4] does not perform well in case of stroke detection.

This paper shows that the proposed preprocessing algorithm allows to: (1) achieve good performance even when the simple DAS algorithm is used; (2) outperform state-ofthe-art preprocessing algorithms; (3) further improve the performance (and/or decrease the number of antennas) by using a multistatic approach with just a modest increase in computational complexity (thus still meeting the requirements in terms of response time).

Results have been achieved by generating noisy backscattered signals via a FDTD (finite-difference timedomain software) 3D dispersive brain model. The head model, the antenna array and the acquisition system of backscattering signals have been simulated using FDTD software. The brain model is a "stratified" model as in [28]. Performance is assessed in terms of accuracy in stroke localization, amount of artifacts not completely removed and computational complexity.

\section{Methods}

\subsection{System model}

A block diagram of the overall system is shown in Fig. 1.

The patient is scanned with a diagnostic device consisting of an UWB antenna array system located around the head, arranged in an anatomical helmet-like structure. Each antenna transmits an UWB pulse (Ricker pulse) into the tissues and collects the backscattered signals, i.e., signals scattered from tissue discontinuities. Two radar approaches are considered: monostatic and multistatic.

The transmitted pulse has a bandwidth that extends from 1 to $4 \mathrm{GHz}$, as a consequence of a good trade-off between radar spatial resolution and waves attenuation inside the human brain [22].

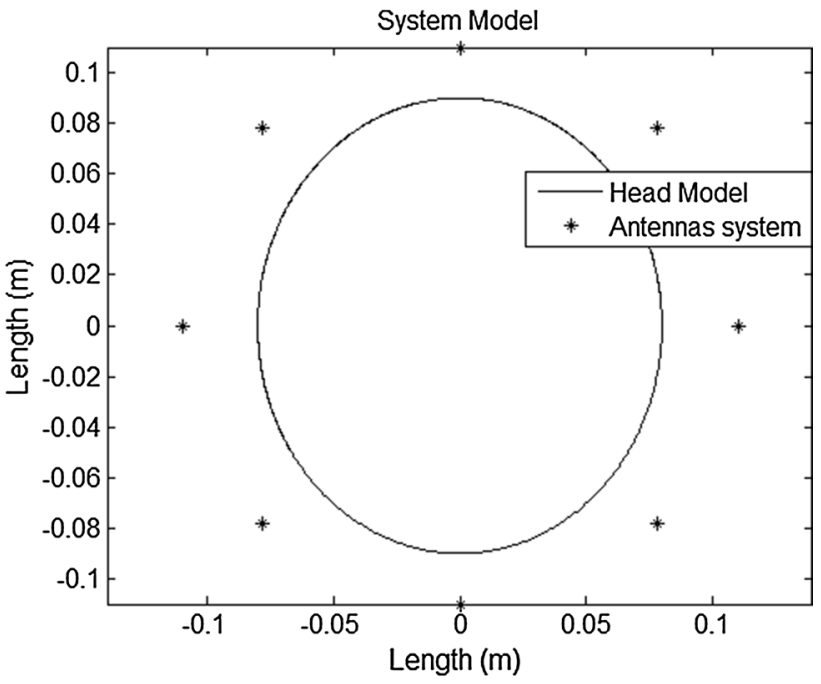

Fig. 2 Head and antenna system model

Table 1 Head tissue properties [3]

\begin{tabular}{llll}
\hline Tissue & Dielectric constant & Loss tangent & $\begin{array}{l}\text { Conductivity } \\
\left(\mathrm{S} \mathrm{m}^{-1}\right)\end{array}$ \\
\hline Skin & 37.952 & 0.28184 & 1.4876 \\
Bone & 11.352 & 0.25597 & 0.40411 \\
White matter & 36.107 & 0.24699 & 1.2403 \\
Blood & 58.181 & 0.31981 & 2.5878 \\
\hline
\end{tabular}

Backscattered signals have been simulated using a finite-difference time-domain (FDTD) software. Backscattered signals are then preprocessed by an artifact removal algorithm. This preprocessing phase is crucial to guarantee good performance when a MWI approach is used. Then, the "cleaned" signals are processed by a beamforming algorithm to build a map of the backscattering energy of the head under examination. The antenna array assembly, the type of signal transmitted by the antennas and the 3D head model are the input data of the FDTD software.

The head has been modeled as a 3D structure, with different layers. The 3D model consists in a blended ellipsoid, simulating the real head size. In Cartesian coordinate system, the planar section of the model has $80 \mathrm{~mm}$ semi-axis along $x$ and a $90 \mathrm{~mm}$ semi-axis along $y$. The depth along $z$ is $100 \mathrm{~mm}$. The head consists of a 2 -mm-thick skin layer, a 6-mm-thick bone layer and brain matter. The dispersive 
properties of the head tissues have been included in the FDTD model, setting different dispersive electrical properties for skin, bone, brain matter and bleedings. The electric properties to take into account the dispersivity of the biological tissues have been evaluated according the ColeCole model. In particular, the calculated parameters are fitted to a second-order polynomial. Figure 2 shows the planar section of the system model, with a planar section of the head as an ellipse and the antenna system as asterisks.

Table 1 reports the main parameters of the different head tissues considered in the model for the computation of the backscattered signals, at center frequency of $2.5 \mathrm{GHz}$ [3].

Our model also considers a coupling medium between the antennas system and the head. The coupling medium is a mixture of different substances with dielectric constants similar to the one of the skin, and it is crucial to ease the wave propagation inside the tissues and reduce the reflection contribution from the head-air interface.

\subsection{Processing phase}

In this paper, the map of backscattering energy of the head is built through a wideband confocal modality based on DAS beamforming. The DAS algorithm applies a beamformer to each return signal from each antenna. The goal is to pass unchanged the signals reflected from one specific point and attenuate the ones coming from any other location. The received signals are time shifted to align them and synthetically focused in a point of the tissue. The signal energy of the focal point is calculated and associated with the intensity of the corresponding pixel. If a scattering object exists (for instance, a lesion) in the focal point, signals are summed coherently and the energy is relatively high. On the other hand, if the lesion is not in the focal point (if the focal point is a healthy tissue), signals are summed incoherently and the energy level is low.

The output $z\left[n, r_{0}\right]$ of the DAS at the $n$th sample of the focal point $r_{0}$ can be written as:

$z\left[n, r_{0}\right]=\sum_{i=1}^{N} x_{i}\left[n-\operatorname{delay}_{i}\left(r_{0}\right)\right]=\sum_{i=1}^{N} x_{i}\left[n-\left(n_{a}-\tau_{i}\left(r_{0}\right)\right)\right]$

where $N$ is the number of channels, $\boldsymbol{x}_{i}$ is the backscattered signal of $i$ th channel, $n_{\alpha}$ is the reference time to which all received signals are aligned and $\tau_{i}\left(r_{0}\right)$ is the roundtrip propagation delay for location $r_{0}$ in the $i$ th channel.

The computational complexity of this processing is low, and this is a desirable characteristic when the final objective is the design of a low-cost and fast-response device. It could be possible to improve the performance by using a more powerful, but also more complex, processing algorithm. As an example of more powerful and complex algorithm, in the comparison results shown later we have

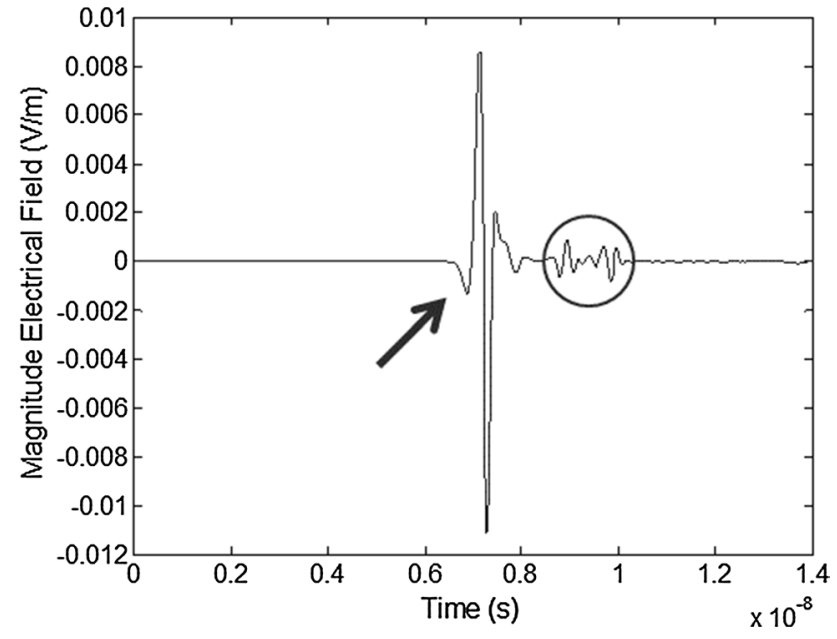

Fig. 3 Received backscattered signal. The arrow shows the reflected signal from skin-coupling medium interface, and the circle shows the stroke contribution

considered the MIST algorithm, proposed for breast cancer in [1] and extended to the stroke detection in [19].

\subsection{Preprocessing phase}

UWB backscattered signals contain a lot of useless information (artifacts), which might lead to wrong diagnostic results. Strong artifacts are due to the reflection from the skin-coupling medium interface. This type of artifacts is highly similar across all channels, and they appear earlier in time than other reflections as the distance between the skin and the antennas is shorter than the distance of any stroke and the antennas. Figure 3 shows an example of the time relation between the signal that is reflected from the skin-coupling medium interface (indicated with an arrow) and the signal that is reflected from the stroke (indicated with a circle). Therefore, this type of artifact can be easily removed by time windowing. However, this time windowing is not enough as other types of artifacts are present.

Figure 4 shows the energy map obtained by processing the backscattered signals with DAS and MIST beamformer, without performing any further preprocessing besides time windowing. The external ellipse is the planar section of the head, while the internal black circumference is the bleeding extension. The energy output is represented as colored pixel in normalized energy scale.

From Fig. 4, it is clear that the signal preprocessing stage is an essential step for a correct bleeding detection.

The following main approaches for artifact removal in the field on MWI can be found in the literature [4]:

Wiener filters: It was originally proposed by Bond [1] and modified in [20] for stroke detection and multistatic 
Fig. 4 Energy maps without the preprocessing phase. The energy maps have been obtained by processing the backscattered signals with DAS and MIST beamformer without the preprocessing phase
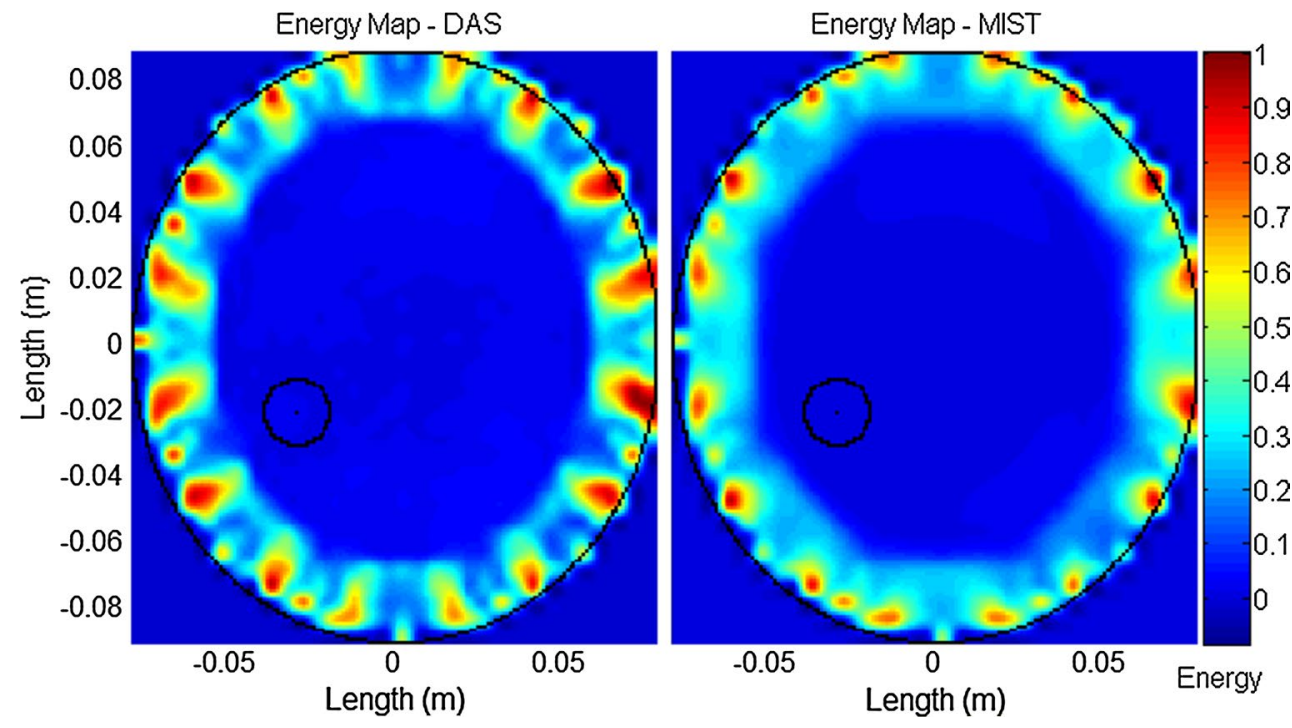

mode. The artifact in each channel is estimated as a filtered combination of the signals in all the other channels. This algorithm needs the a priori knowledge of the time interval in which the backscattered signals contain only the artifact contribution. In addition, it introduces a small level of distortion in the lesion contribution and it is computationally complex.

PLSR: It is based on statistical regression techniques [20]. The artifact on each channel is estimated as a linear combination of the artifact in all other channels, utilizing the maximization of correlation as constraint.

Entropy-based time windowing [29]: the algorithm applies time windowing to the received signals to get rid of the artifacts. This window is designed according to the entropy of the signals at each time instant, in order to eliminate the similar part of the data. Entropy is a measure of the uncertainty of a variable and can be seen as a measure of the signal variation. For example, a large entropy value occurs when data are very similar. This algorithm requires no a priori information and brings no distortion to the useful data. However, as reported in [4], it often fails to accurately estimate the exact portion of signals containing the artifacts.

SVD_singular value decomposition: SVD has been used for clutter reduction in GPR (ground-penetrating radar) and through-wall imaging [26]. Moreover, it has been considered in the comparison performed in [4] for breast cancer. This algorithm is tightly related to the algorithm here proposed. The objective is to decompose the received signals into artifact and useful data subspace. Only the subspace containing useful information is selected, and artifacts are discarded.

Head symmetry [15]: The artifacts are removed by subtracting the backscattered signals at antenna that face each other in the array with respect to the head midline, i.e., the central line that divides the head into left and right hemisphere.

In the following, both monostatic and multistatic modes are considered. In the monostatic mode, each antenna is activated in turn, transmitting a broadband pulse and receiving the backscattered signals to be processed. Let $\boldsymbol{x}_{\boldsymbol{i} i}$ be the monostatic signals, when the $i$ th antenna transmits and the $i$ th antenna receives. Considering $N$ antennas, the number of monostatic channels is $C=N$. In the multistatic mode, each antenna transmits a broadband pulse and all the antennas, including the transmitter, receive the backscattered signals. Considering $N$ antennas, the number of multistatic channels is $C=N^{*} N$. However, not all signals carry useful information. For example, channels related to antennas that are not in visibility, since the head is interposed between them, do not contain relevant information. On the other hand, channels related to adjacent antennas have very high information content. Let $\boldsymbol{x}_{\boldsymbol{j} i}$ be the signal associated to the jith channel, in which the $i$ th antenna transmits and the $j$ th antenna receives. The monostatic signals are those ones with $j=i$. The selected multistatic signals are those ones with $j=i \pm 1$, i.e., only the channels associated with adjacent antennas. Considering $N$ antennas, the number of selected multistatic channels is $C=2 * N$. Hence, two groups of channels are defined, the monostatic channels $(j=i)$ and the selected multistatic channels $(j=i \pm 1)$, with $C=N+2 * N$ channels. Only those signal groups are preprocessed and processed. The signals within the same group are similar, since the channels into the group have similar geometric characteristics.

Before passing the backscattered signals to the preprocessing algorithm, a first cleaning of the signal is performed by a simple low-pass filter [20]. Figure 5 shows the spectrum of the backscattered signal in presence of artifact 


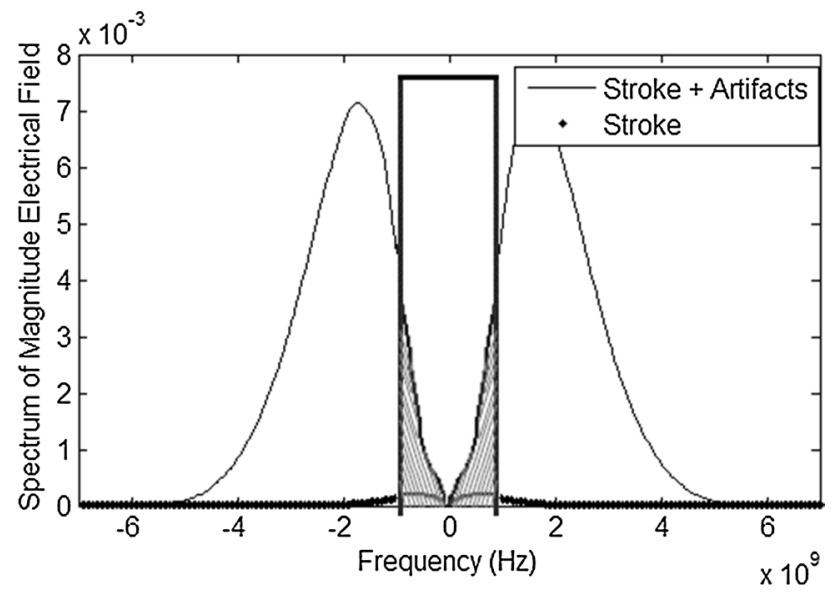

Fig. 5 Backscattered signal spectrum. The solid line represents the spectrum of received signal with artifacts contribution and stroke contribution. The dotted line represents the spectrum of ideal received signal, without artifacts

(solid line) and the spectrum of the received signal when there are no artifacts (dotted line).

It is evident that the stroke contribution is mainly localized in the low frequencies of the spectrum. Therefore, a low-pass filtering has been performed, shown in Fig. 5 as a rect, which removes the high frequency part of the spectrum. The cutoff frequency of the filter has been empirically selected. It has been verified that this preliminary "cleaning" of the signal significantly improves the performance for all the preprocessing algorithms that have been considered. Nevertheless, it does not remove the main part of the artifacts that are within the bandwidth of the lowpass filter. Therefore, artifact removal is still needed.

\subsection{Proposed preprocessing algorithm-PCA (Principal Component Analysis)}

The proposed preprocessing algorithm is based on the observation that the artifacts in each group of channels are highly correlated. This is due to the rather symmetrical shape of the head and of the antenna setup. On the other hand, the stroke contribution to the reflected signal of each channel is uncorrelated, since it occurs in different time intervals and follows different propagation paths.

PCA is a statistical technique widely used in pattern recognition and most generally in data analysis problems $[10,26]$. It is mainly used to find and emphasize correlation between features and perform a dimensionality reduction by projecting to a low-dimensional subspace. PCA allows to find a set of mutually orthogonal directions, called principal axes, which represent directions along which the variance in the observed data is maximized. The projections of the original variables along the principal directions are called principal components (PC) or scores. PCA returns principal directions ordered by decreasing variance, so the first components capture the highly correlated data, while the last ones represent the uncorrelated information. Applying PCA to our low-pass filtered signals allows to decompose the data into two subspaces: the correlated subspace (first PCs), which mainly contains the artifacts contribution, and the uncorrelated subspace (last PCs), which capture the stroke reflections.

Let us define $X$ as a $[\operatorname{tr} x C]$ matrix, where each column $\boldsymbol{x}_{\boldsymbol{j}}$, with $j=1, \ldots, C$, is a vector that contains the samples of low-pass filtered signal of the $j$ th channel. $t r$ is the number of time samples, and $C$ is the number of similar channels considered. The PCA linear transformation is applied to $\bar{X}=X-\operatorname{mean}(X)$ :

$Y=\bar{X} V$

$V$ is a $[C \times C]$ linear projection matrix in which the $i$ th column is called $i$ th principal direction and is equal to the $i$ th eigenvector of the covariance matrix of $\bar{X}$. The eigenvectors are sorted by decreasing eigenvalues $\sigma$. An eigenvalue is a kind of "measure" of the signal variance captured by the corresponding eigenvector. $Y$ is a $[\operatorname{tr} x C]$ matrix that represents the signals projected in the principal components space. The $i$ th column of $Y$ corresponds to the projection of $\bar{X}$ along the $i$ th principal direction and is called $i$ th principal component score.

The original data matrix can be reconstructed by a linear combination of the principal directions (using orthogonality of $V$ ) as follows:

$\bar{X}=Y V^{T}=\sum_{i=1}^{C} y_{i} v_{i}^{T}=\sum_{i=1}^{k-1} y_{i} v_{i}^{T}+\sum_{i=k}^{C} y_{i} v_{i}^{T}$

where $\boldsymbol{y}_{\boldsymbol{i}}$ is the $i$ th principal component, and $\boldsymbol{v}_{\boldsymbol{i}}$ is the $i$ th principal axis. As previously mentioned, the first terms of the sum, until $k-1$, represent the highly correlated data in $\bar{X}$ (i.e., the artifacts), while the last components contain the uncorrelated part, including the stroke contribution. Therefore, a new observation matrix is constructed according to (4).

$\bar{X}_{\mathrm{rec}}=\sum_{i=k}^{C} y_{i} v_{i}^{T}$

The eigenvalue $k$ is chosen as the nearest to the mean value of all the eigenvalues $\sigma$, i.e.,

$k=\underset{i}{\arg \min }\left(\left|\sigma_{i}-\sigma_{\text {mean }}\right|\right)$

In the algorithm considered in [4] for breast cancer, the same decomposition between correlated part of the signal and uncorrelated part of the signal is implemented by doing 
the SVD directly to the matrix $X$. Moreover, the eigenvalue $k$ is selected according to:

$$
k=\underset{i}{\arg \max }\left(\sigma_{i}-\sigma_{i+1}\right)
$$

\section{Results}

In this section, first of all the proposed PCA-based preprocessing algorithm is compared with state-of-theart algorithms presented in the previous section, both in a monostatic and in a multistatic modes. Signals are first passed through a low-pass filter, and then the DAS algorithm is used to build the energy map. Backscattered signals are generated by a FDTD using a 3D head model and eight transmitting/receiving antennas. Noisy backscattered signals have been generated by adding white Gaussian noise with a SNR of $10 \mathrm{~dB}$. The performance has been assessed considering a spherical bleeding (hemorrhage) with a diameter of $20 \mathrm{~mm}$. Two different positions of the bleeding are considered (usually, the hemorrhages close to the scalp are more difficult to localize as very close to the main artifact).

Let us denote with $S$ the set of points that form a 2D window around the detected target in the head and $H$ the
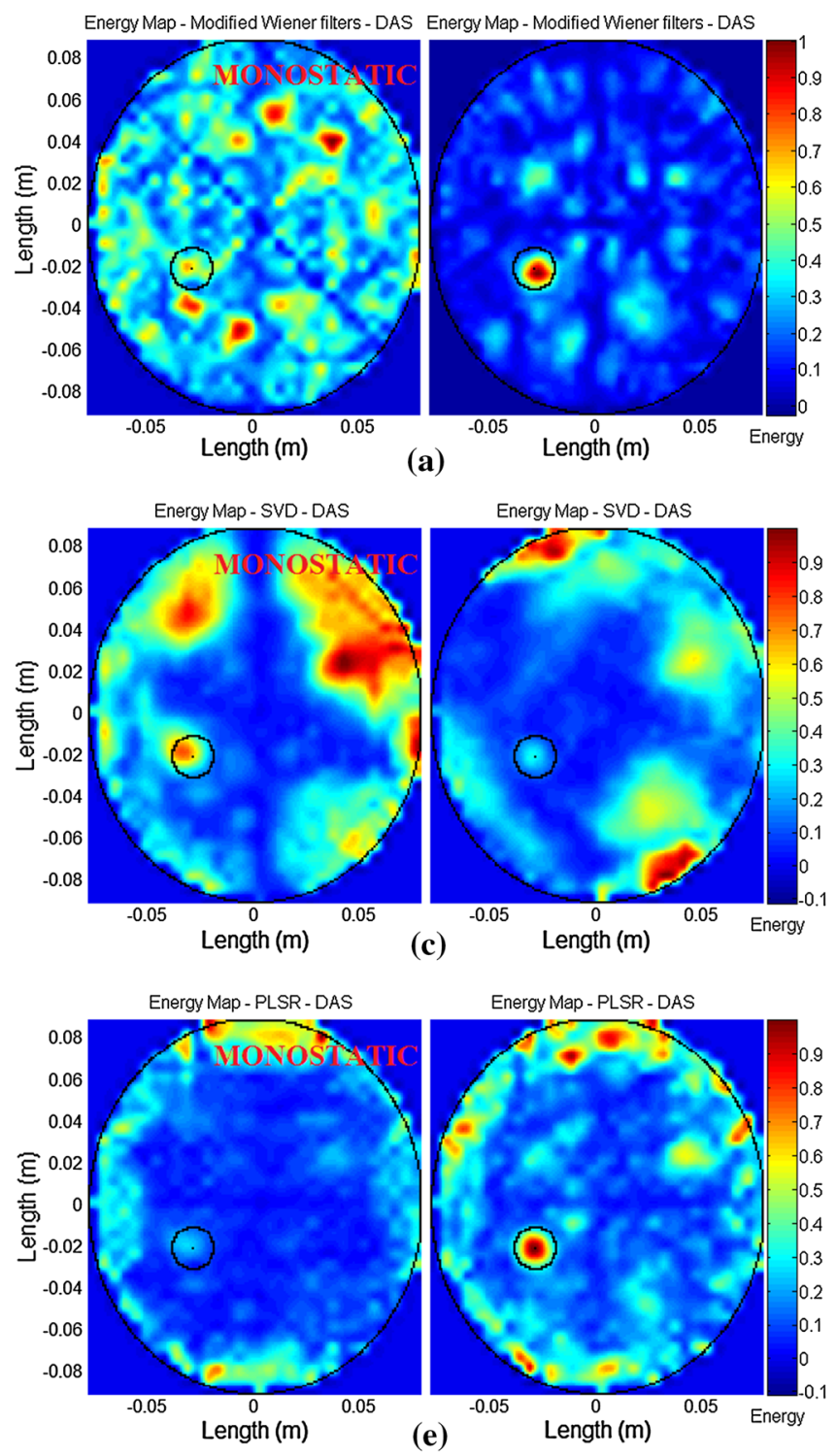

Fig. 6 Energy maps for different preprocessing algorithms for stroke location 1 . The energy maps have been obtained with the monostatic and multistatic DAS beamformer and the artifact removal algorithm
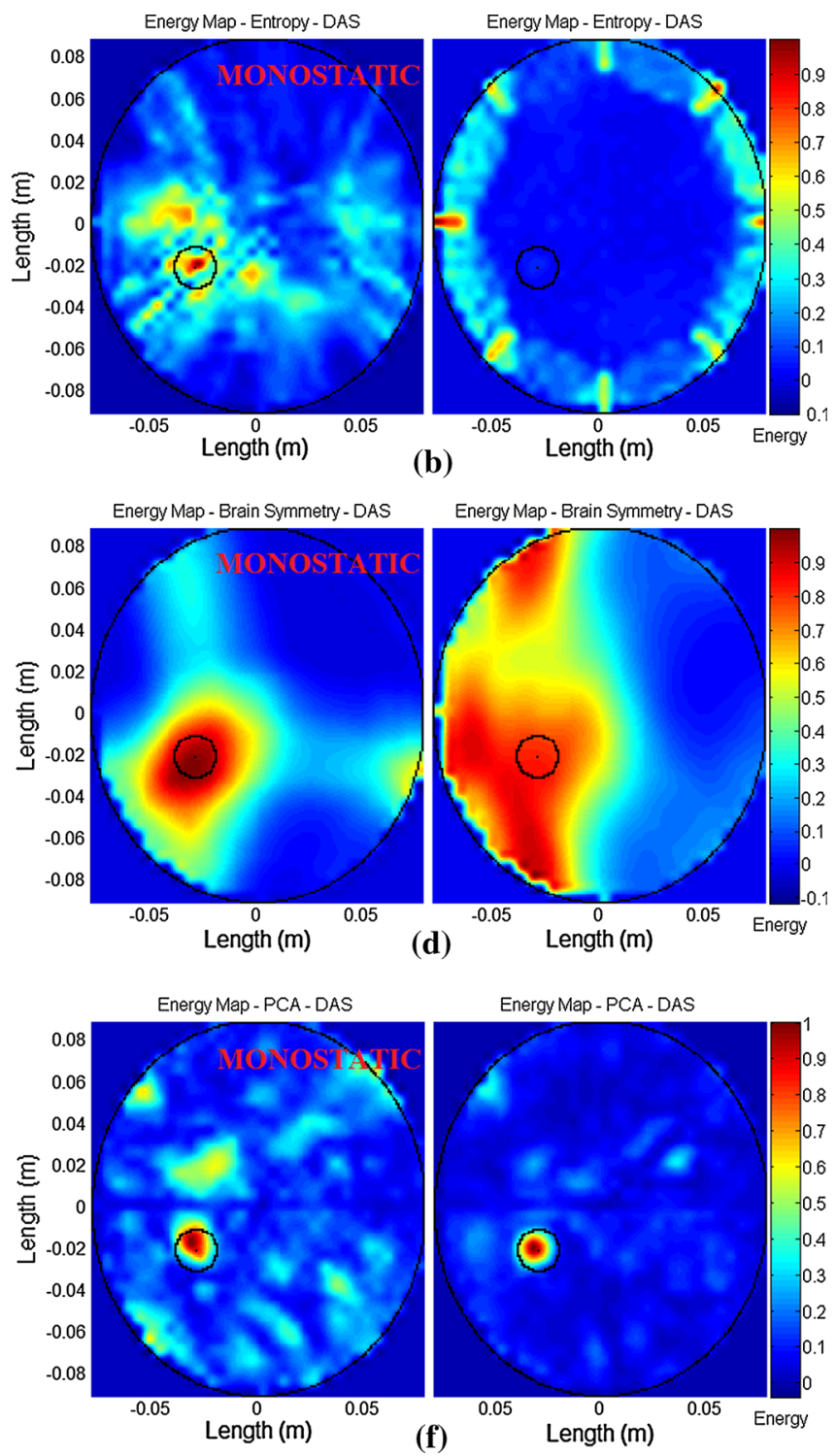

based on modified Wiener filters (a), entropy-based time windowing (b), SVD (c), brain symmetry (d), PLSR (e) and PCA (f) 
set of all points within the head area. The following commonly used metrics have been selected to compare the performance of different algorithms:

The absolute distance $\Delta$ between the real center of the stroke $\chi$ and the location of the stroke estimated as the point with maximum intensity in the energy map distribution $I$ :

$\Delta=\left\|p^{*}-\chi\right\|$

where

$$
p^{*}=\underset{p \in H}{\arg \max }[I(p)]
$$
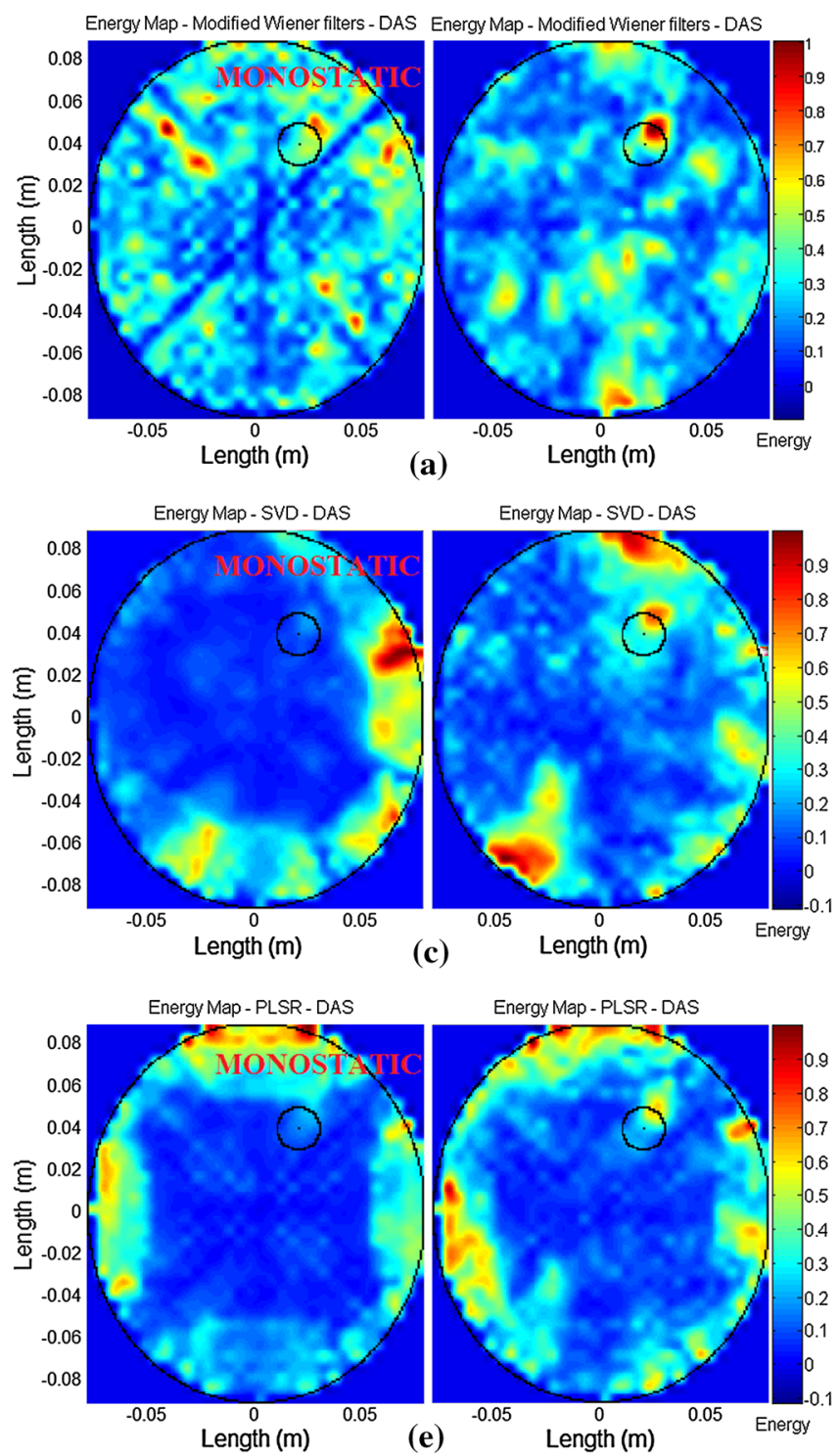

Fig. 7 Energy maps for different preprocessing algorithms for stroke location 2 . The energy maps have been obtained with the monostatic and multistatic DAS beamformer and the artifact removal algorithm
The stroke is localized with more precision when this distance is lower.

The metric $Q$ is defined as the ratio of the average intensity in the actual stroke area to the average intensity in the rest of head tissues, given by:

$Q=\frac{\operatorname{mean}[I(p)]}{\operatorname{mean}[I(p)]} \quad \forall p \in H \& p \notin S$

A high value of this metric implies that stroke intensity is stronger than the intensity of the background region, and hence that there is a low amount of artifacts not completely removed.
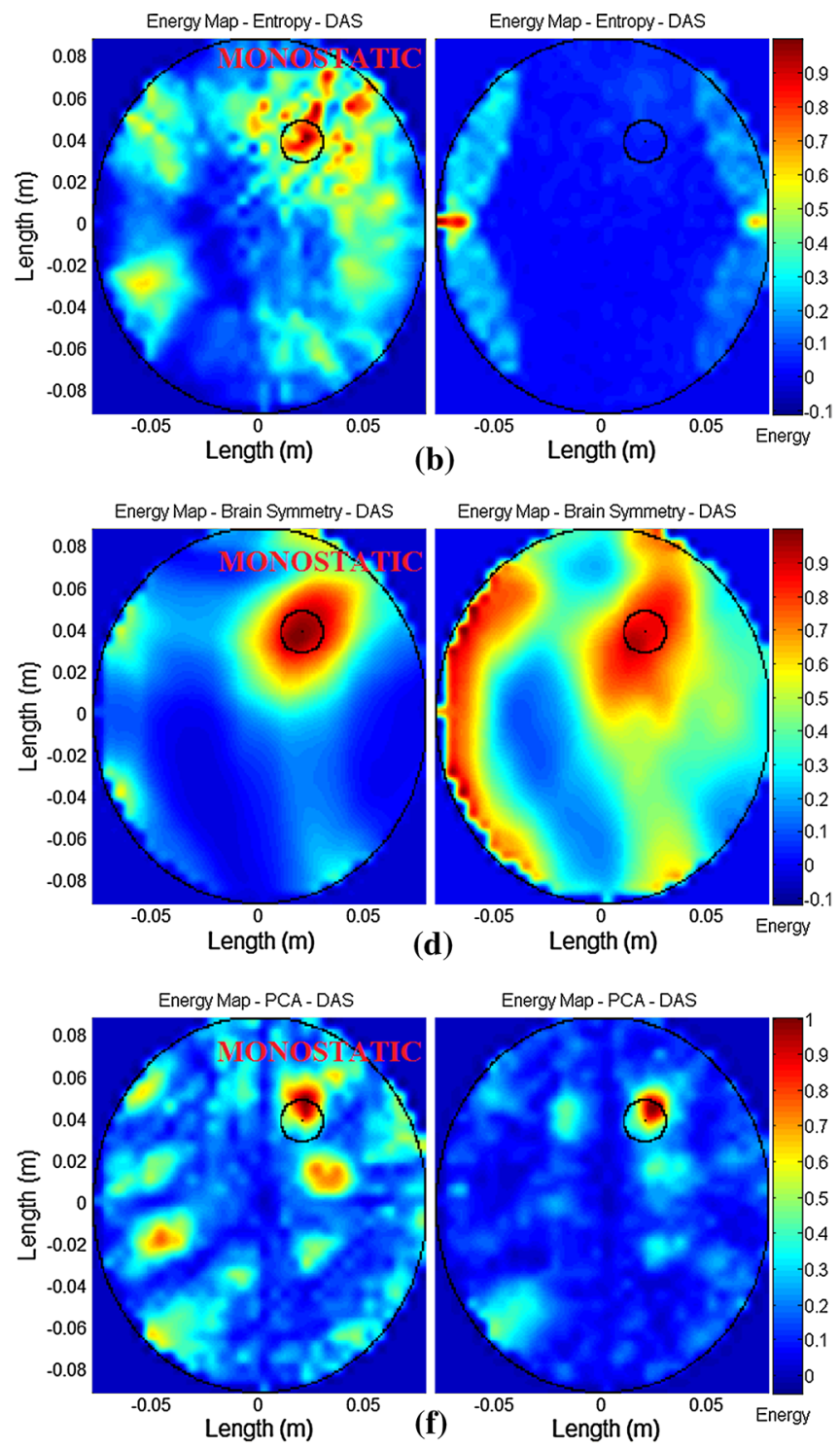

based on modified Wiener filters (a), entropy-based time windowing (b), SVD (c), brain symmetry (d), PLSR (e) and PCA (f) 
The signal-to-clutter ratio $(S C R)$ is used to evaluate how much energy of the stroke area is above the energy of the clutter, which can be written as follows:

$$
\mathrm{SCR}=\frac{\text { mean }\left[I(p)_{\text {stroke }}\right]}{\text { mean }\left[I(p)_{\text {clutter }}\right]} \quad \begin{aligned}
& \forall p \in S \\
& \forall p \in S
\end{aligned}
$$

where $I(p)_{\text {stroke }}$ is the energy value in the $2 \mathrm{D}$ window $S$ around the stroke, and $I(p)_{\text {clutter }}$ is the energy value over the same region $S$ when the stroke is not present. Clutter is due to the residual artifacts, and the mean energy value of clutter is calculated in a stroke-free model, i.e., healthy head model without stroke. If artifacts are not effectively removed, the SCR will be low.

As already stated, in this work, another important performance metric is the computational complexity, which has been evaluated as the order of magnitude of the number of basic mathematic operations of each algorithm.

Figures 6, 7 show the backscattering energy map of a planar section of the head under examination for two positions of the stroke, using DAS and different preprocessing algorithms. Figures on the left refer to the monostatic mode and figures on the right to the multistatic mode. The external ellipse is the planar section of the head. The internal black circle is the bleeding area. The energy output is represented as colored pixel in a normalized energy scale.

Tables 2 and 3 show the same comparisons in terms of the metrics previously introduced.

Table 2-Metrics performance related to Figs. 6 and 7 for monostatic DAS.

Table 3-Metrics performance related to Fig. 6 and 7 for multistatic DAS.

Table 2 Metrics performance related to Figs. 6 and 7 for monostatic DAS

\begin{tabular}{lcrr}
\hline & $\Delta(\mathrm{mm})$ & $Q(\mathrm{~dB})$ & $\mathrm{SCR}(\mathrm{dB})$ \\
\hline Stroke location 1 & & & \\
Modified Wiener filters & 88.459 & 0.590 & 8.048 \\
Entropy & $2.761 \times 10^{-4}$ & 7.244 & 10.064 \\
SVD & 83.216 & -0.872 & -3.527 \\
Brain symmetry & 7.070 & 12.201 & 32.963 \\
PLSR & 116.619 & -3.806 & 9.858 \\
PCA & 4.999 & 4.892 & 12.857 \\
Stroke location 2 & & & \\
Modified Wiener filters & 65.191 & 1.260 & 6.561 \\
Entropy & $2.761 \times 10^{-4}$ & 6.035 & 6.444 \\
SVD & 50.990 & -6.956 & 7.712 \\
Brain symmetry & 7.070 & 12.571 & 30.728 \\
PLSR & 45.000 & -3.410 & 24.215 \\
PCA & 5.000 & 5.211 & 5.561 \\
\hline
\end{tabular}

Table 3 Metrics performance related to Figs. 6 and 7 for multistatic DAS

\begin{tabular}{lrrr}
\hline & $\Delta(\mathrm{mm})$ & $Q(\mathrm{~dB})$ & $\mathrm{SCR}(\mathrm{dB})$ \\
\hline Stroke location 1 & & & \\
Modified Wiener filters & 4.999 & 5.979 & 11.029 \\
Entropy & 53.851 & -13.055 & -8.050 \\
SVD & 84.852 & -7.096 & -4.139 \\
Brain symmetry & 50.249 & 6.632 & 20.158 \\
PLSR & 91.241 & 1.479 & 14.550 \\
PCA & 4.999 & 9.252 & 16.906 \\
Stroke location 2 & & & \\
Modified Wiener filters & 5.000 & 3.878 & 3.824 \\
Entropy & 107.703 & -3.303 & -4.749 \\
SVD & 46.098 & 3.633 & -0.496 \\
Brain symmetry & 11.180 & 5.327 & 28.126 \\
PLSR & 68.007 & -0.406 & 19.906 \\
PCA & 5.000 & 8.530 & 11.939 \\
\hline
\end{tabular}

Table 4 Preprocessing algorithms computational complexity

\begin{tabular}{lllllll}
\hline & $\begin{array}{l}\text { Modified } \\
\text { Wiener } \\
\text { filters }\end{array}$ & Entropy & SVD & $\begin{array}{l}\text { Brain sym- } \\
\text { metry }\end{array}$ & PLSR PCA \\
\hline Complexity & $10^{10}$ & $10^{5}$ & $10^{8}$ & $10^{4}$ & $10^{5}$ & $10^{8}$ \\
\hline
\end{tabular}

First of all, comparisons show that both for the monostatic and for the multistatic modes and with a moderate number of antennas (8 rather than 16-32 as considered in [13]), the proposed preprocessing algorithm shows better performance. In the monostatic mode, the entropy-based algorithm and the symmetry-based algorithm have metrics comparable with the ones achieved with the PCA-based algorithm. However, as shown in Figs. 6, 7, the entropybased algorithm introduces false alarms, and the symmetry-based one is less accurate in the definition of the lesion contours and its extension.

For a monostatic system, the performance difference is still not negligible. The distance $\Delta$ in the case of monostatic DAS is 4.999 for the PCA-based algorithm and 88.459 for the algorithm based on Wiener filters. The $Q$ is 4.892 and 0.590 , respectively. On the other hand, in terms of complexity (see Table 4), the Wiener filtering is much more complex $\left(10^{10}\right.$ versus $10^{8}$ for PCA-based algorithm). Specifically, the run time of the algorithms has been measured on a laptop with Intel Core i3 M330 $2.13 \mathrm{GHz}$ CPU. Several runs have been performed of all algorithms, and the average run time has been calculated. In particular, for Wiener filtering, the average run time is about $450 \mathrm{~s}$, whereas for the PCA-based algorithm the average run time is about $2 \mathrm{~s}$. 

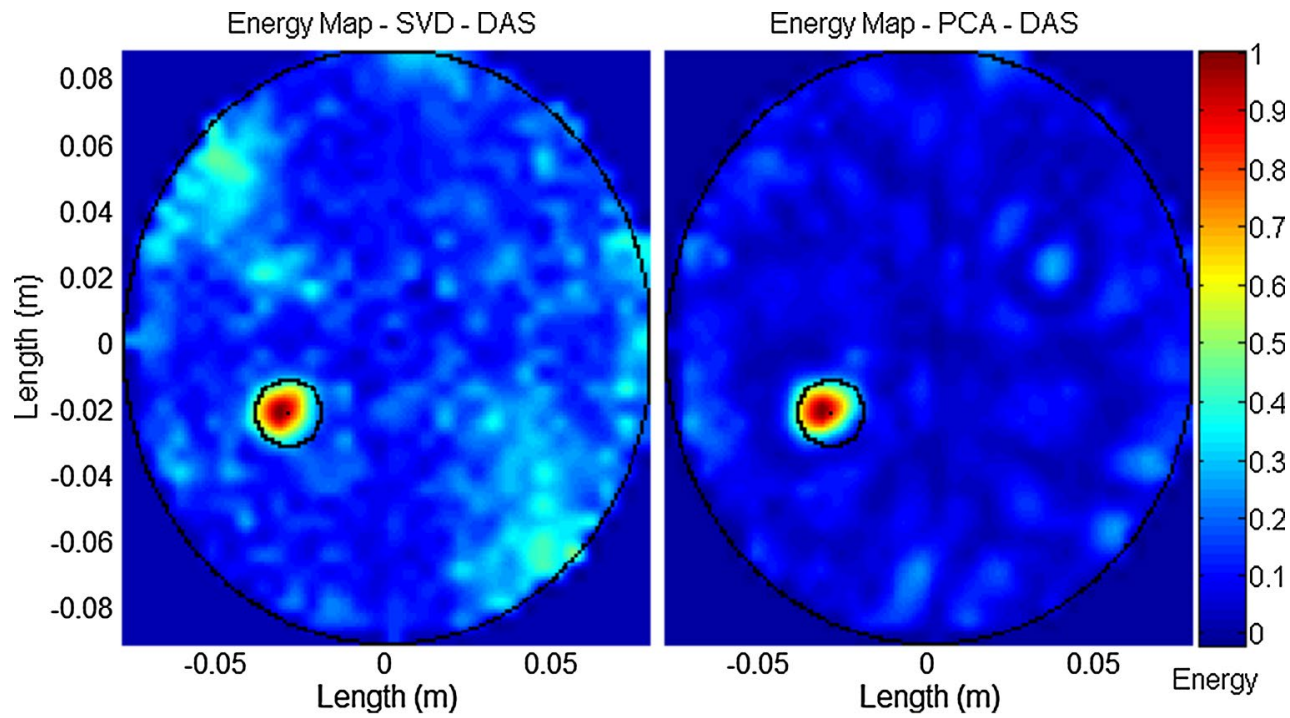

Fig. 8 Energy maps for signals with $20 \mathrm{~dB}$ of SNR. The energy maps have been obtained with the multistatic DAS beamformer and the artifact removal algorithm based on SVD, on the left, and PCA, on the right, when the signals have a SNR of $20 \mathrm{~dB}$
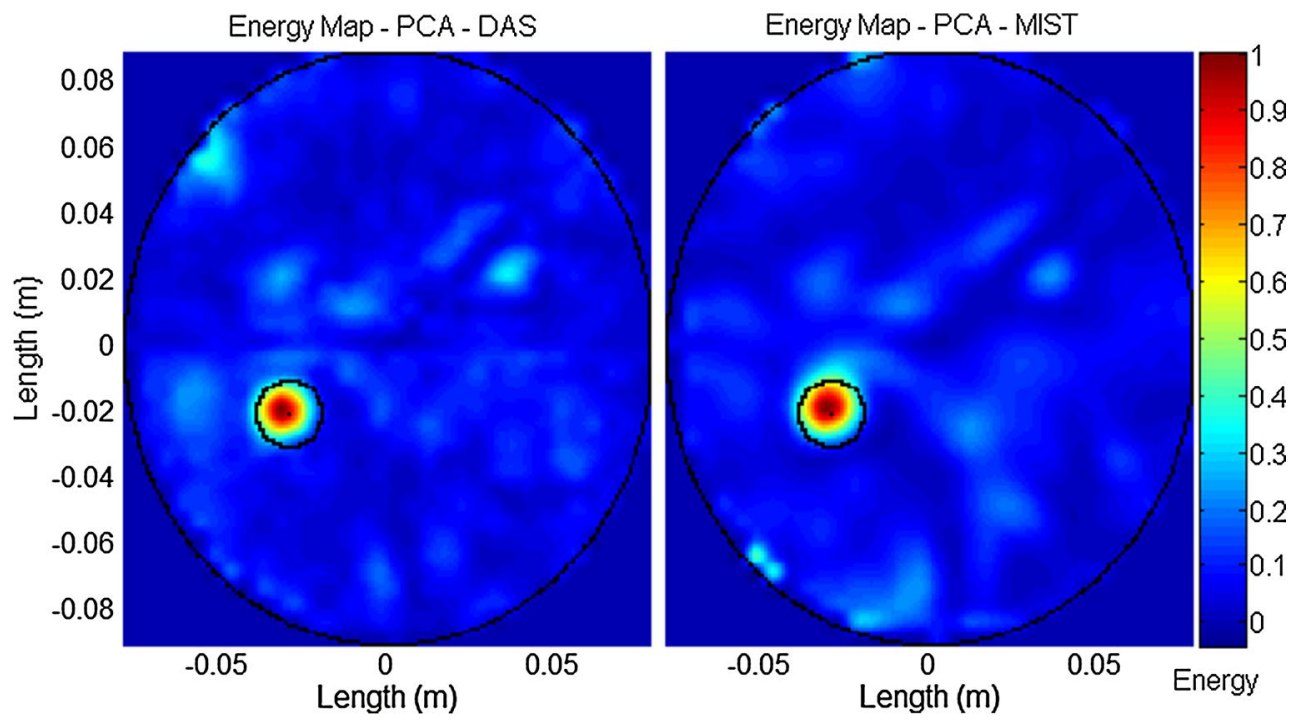

Fig. 9 Energy maps with DAS and MIST beamformer. The energy maps have been obtained with the proposed preprocessing algorithm based on PCA and applied to DAS, on the left, and MIST, on the right, beamformer

It is also worth noting that some of the state-of-the-art algorithms do not properly work in case of a multistatic system. This is the case for the entropy-based algorithm, the SVD algorithm and the symmetry-based algorithm. This is probably related to the fact that they are not much robust to noise and a multistatic system amplifies this behavior as more channels are processed and also more noise is collected. As a matter of fact, these results change significantly if a lower level of noise is considered. For instance, in Fig. 8, performance of the SVD with a SNR of $20 \mathrm{~dB}$ is considered. However, even if its performance is greatly improved, still the PCA-based algorithm has better performance.

Finally, we have also compared the performance of the DAS beamformer with the more complex MIST beamformer when the PCA preprocessing is used. Figure 9 displays the energy maps, and Table 5 summarizes the metrics.

As expected, the MIST algorithm shows slightly better performance, with the same accuracy in stroke localization but improved reduction of the artifacts, as shown by the distance $\Delta$ and the $Q$. However, as shown in Table 5 , the MIST has the not negligible disadvantage of increasing the 
Table 5 Metrics performance related to Fig. 9

\begin{tabular}{lll}
\hline & DAS & MIST \\
\hline$\Delta(\mathrm{mm})$ & 4.999 & 4.999 \\
$Q(\mathrm{~dB})$ & 9.252 & 10.680 \\
$\mathrm{SCR}(\mathrm{dB})$ & 16.906 & 20.286 \\
Beamforming complexity & $10^{4}$ & $10^{9}$ \\
\hline
\end{tabular}

computational complexity, especially in case of multistatic mode and thus increasing the response time of the diagnostic device. The MIST algorithm complexity is of 5 orders of magnitude greater than the one of the DAS. To give an idea, using a laptop with Intel Core i3 M330 $2.13 \mathrm{GHz}$ CPU, the MIST beamformer run time is of some hours of processing (about 14,000 s), while the DAS needs only few minutes (about $205 \mathrm{~s}$ ).

Therefore, the proposed preprocessing algorithm has the important advantage of achieving performance that is comparable or just slightly worse than the one of a more powerful processing algorithm, with a negligible increase in computational complexity.

\section{Discussion}

This paper addresses the use of UWB radar imaging for stroke detection. A PCA-based artifact removal algorithm has been proposed, which has the important advantage of greatly improving the performance of a low complexity beamformer, such as the DAS algorithm, in terms of stroke localization capability and amount of artifacts not completely removed. Simulation results prove that the DAS algorithm combined with the proposed PCA-based preprocessing algorithm performs significantly better compared with state-of-the-art preprocessing algorithms, both in monostatic and in multistatic modes. Only in the multistatic mode, the Wiener filtering preprocessing algorithm has similar performance, but at expense of much higher computational complexity. Moreover, it has been shown that the combination of the proposed algorithm with the DAS algorithm has performance just slightly worse than the ones of the combination of the same preprocessing algorithm and the more complex MIST beamformer. Another novel element of this paper is that we consider both a monostatic and a multistatic cases. Most of the works for stroke detection are only limited to the monostatic case. The multistatic approach has the advantage of providing more accurate performance, as the number of channels is higher, at expense of higher complexity (we have to process more data). However, by applying the PCA-based preprocessing algorithm is possible to achieve good performance with the low complex DAS beamforming algorithm, and hence, the further improvement in performance achievable by using a multistatic rather than a monostatic mode comes at a lower cost in terms of complexity.

\section{Conclusion}

Transportability, low cost and short response time are key requirements for a novel diagnostic device for stroke detection, based on microwave UWB radar imaging, which could play an important role in emergency scenarios. These requirements pose challenges to the signal processing algorithms that must guarantee good performance while keeping low the number of antennas and computational complexity (e.g., the response time of the device). This paper proposes a PCA-based preprocessing algorithm. Shown results indicate that the proposed algorithm allows the implementation of a UWB radar imaging device that can use: simple DAS processing; a low number of antennas; a multistatic approach at low cost in terms of computational complexity increases; and it is able to achieve improved performance with respect to the state-of-the-art solutions.

The next step before going for clinical trials is the implementation of a realistic 3D head phantom, with electrical properties that emulate those of real tissues. This step is fundamental to take into account the less "regular" and not perfectly symmetrical head structure, which are expected to have an impact on the performance. On the other hand, the results of this paper are fundamental to prove the feasibility of a device characterized by a fast response and low complexity, which are key requirements for such a device and the main motivation for the use of UWB radar imaging for stroke detection.

\section{Compliance with ethical standards}

Conflict of interest The authors declare that they have no conflict of interest.

\section{References}

1. Bond EJ, Li X, Hagness SC, Van Veen BD (2003) Microwave imaging via space-time beamforming for early detection of breast cancer. IEEE Trans Antennas Propag 51(8):1690-1750

2. Caorsi S, Frattoni A, Gragnani GL, Nortino E, Pastorino M (1991) Numerical algorithm for dielectric-permittivity microwave imaging of inhomogeneous biological bodies. Med Biol Eng Compu 29(6):NSS37-NSS44

3. Dielectric properties of body tissue in the frequency range $10 \mathrm{~Hz}-100 \mathrm{GHz}$. http://niremf.ifac.cnr.it/tissprop/. Accessed 10 Oct 2013

4. Elahi MA, Glavin M, Jones E, O'Halloran M (2013) Artifact removal algorithms for microwave imaging of the breast. Prog Electromagn Res 141:185-200

5. Feigin VL, Lawes CM, Bennett DA, Barker-Collo SL, Parag V (2009) Worldwide stroke incidence and early case fatality 
reported in 56 population-based studies: a systematic review. Lancet Neurol 8:355-369

6. Fhager A, Persson M (2012) Stroke detection and diagnosis with a microwave helmet. In: 6th European conference on antennas and propagation (EuCAP). 1796-1798

7. Irastorza RM, Blangino E, Carlevaro CM, Vericat F (2014) Modeling of the dielectric properties of trabecular bone samples at microwave frequency. Med Biol Eng Compu 52(5):439-447

8. Ireland D, Bialkowski M (2011) Microwave head imaging for stroke detection. Prog Electromagn Res M 21:163-175

9. Jauch et al (2013) Stroke 44:870-947

10. Kabourek V, Cerny P, Mazanek M (2012) Clutter reduction based on Principal Component Analysis technique for hidden detection. Radio Eng 21(1):464-470

11. Li X, Hagness S (2001) Confocal microwave imaging algorithm for breast cancer detection. IEEE Microw Wirel Compon Lett 11(3):130-132

12. Medfield Diagnostic (2014) http://www.medfielddiagnostics. com/en/products/. Accessed 12 Nov 2014

13. Mohammed BJ, Abbosh AM, Mustafa S, Ireland D (2014) Microwave system for head imaging. IEEE Trans Instrum Meas 63(1):117-123

14. Mozaffrian D, Benjamin EJ, Go AS et al (2015) Heart disease and stroke statistics-2015 update: a report from American Heart Association. Circulation 131:e29-e322

15. Mustafa S, Mohammed B, Abbosh A (2013) Novel preprocessing techniques for accurate microwave imaging of human brain. IEEE Antennas Wirel Propag Lett 12:460-463

16. O'Halloran M, Jones E, Glavin M (2010) Quasi-multistaitc MIST beamforming for early detection of breast cancer. IEEE Trans Biomed Eng 57(4):830-840

17. Paulson CN, Chang JT, Romero CE, Watson J, Pearce FJ, Levin N (2005) Ultra-wideband radar methods and techniques of medical sensing and imaging. SPIE Smart Med and Biomed Sensor Technol III 6007:96-107

18. Persson M, Fhager A, Trefnà HD, Yinan Yu, McKelvey T, Pegenius $\mathrm{G}$ et al (2014) Microwave-based stroke diagnosis making global prehospital thrombolytic treatment possible. IEEE Trans Biomed Eng 61(11):2806-2817

19. Ricci E, Maggio F, Rossi T, Cianca E, Ruggieri M (2015) UWB radar imaging based on space-time beamforming for stroke detection. In: 6th European conference of the international federation for medical and biological engineering. Springer International Publishing. 946-949

20. Ricci E, Di Domenico S, Cianca E, Rossi T (2015) Artifact removal algorithms for stroke detection using a multistatic MIST beamforming algorithm. In: 37th annual international conference of the IEEE engineering in medicine and biology society EMBC. 1930-1933

21. Ricci E, Colucciello A, Di Domenico S, Cianca E, Rossi T (2015) Modified RAR and PLSR-based artifact removal for stroke detection in UWB radar imaging. In: 5th international conference wireless vitae. in press

22. Scapaticci R, Di Donato L, Catapano I, Crocco L (2012) A feasibility study on microwave imaging for brain stroke monitoring. Prog Electromagn Research B 40:305-324
23. Semenov SY, Corfield DR (2008) Microwave tomography for brain imaging: feasibility assessment for stroke detection. Int $\mathbf{J}$ Antennas Propag. doi:10.1155/2008/254830

24. Semenov SY, Svenson RH, Posukh VG, Nazarov AG, Sizov YE, Bulyshev AE et al (2002) Dielectrical spectroscopy of canine myocardium during acute ischemia and hypoxia at frequency spectrum from $100 \mathrm{kHz}$ to $6 \mathrm{GHz}$. IEEE Trans Med Imaging 21(6):703-707

25. Suzuki S, Matsui T, Kawahara H, Ichiki H, Shimizu J, Kondo $\mathrm{Y}$ et al (2009) A non-contact vital sign monitoring system for ambulances using dual-frequency microwave radars. Med Biol Eng Compu 47(1):101-105

26. Verma PK, Gaikwad AN, Singh D, Nigam MJ (2009) Analysis of clutter reduction techniques for trough wall imaging in UWB range. Prog Electromagn Res B 17:29-48

27. Yin T, Ali FH, Reyes-Aldasoro CC (2015) A robust and artifact resistant algorithm of ultrawideband imaging system for breast cancer detection. IEEE Trans Biomed Eng 62(6):1514-1525

28. Zhang H, Flynn B, Erdogan AT, Arslan T (2012) Microwave imaging for brain tumor detection using an UWB Vivaldi antenna array. Antennas Propag Conf LAPC. doi:10.1109/ LAPC.2012.6402964

29. Zhi H, Chin F (2006) Entropy-based time window for artifact removal in UWB imaging of breast cancer detection. IEEE Signal Process Lett 13(10):585-588

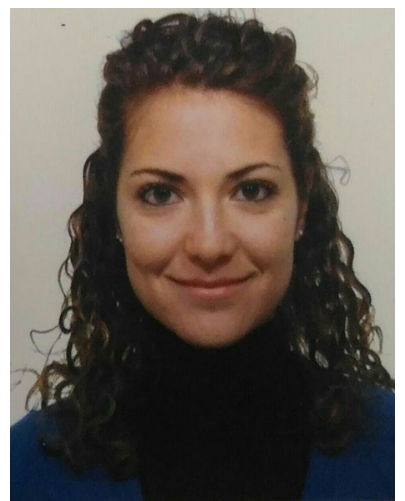

Elisa Ricci received her M.Sc. degree in Medical Engineering in 2012 at University of Rome Tor Vergata and she is postdoc in the Department of Electronic Engineering of the same University. Her research is focused on microwave imaging and UWB radar for biomedical application.

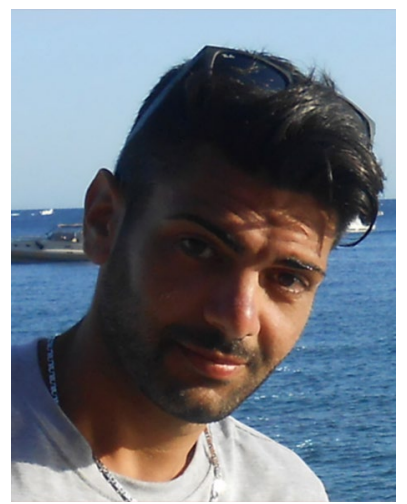

Simone di Domenico received the M.Sc. degree in Internet Engineering from the University of Rome Tor Vergata. He is a Ph.D. student in the Department of Electronic Engineering, and his research is focused on signal processing applied to devicefree human activity recognition. 


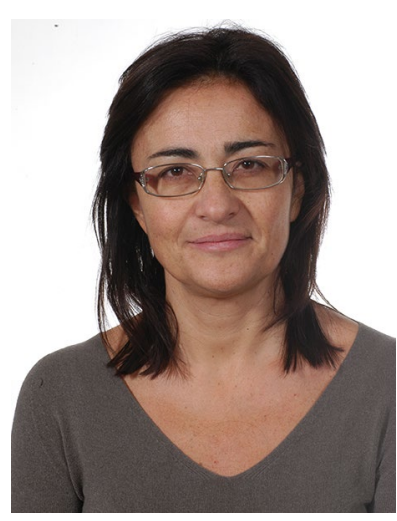

Ernestina Cianca is assistant professor at the Department of Electronic Engineering of the University of Rome Tor Vergata. Her research activity is focused on DSP and beamforming for radar and wireless communications.

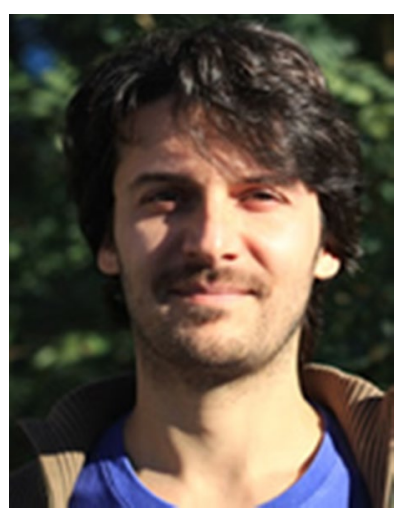

Tommaso Rossi is Assistant Professor at the University of Rome Tor Vergata. His research activity is focused on space systems, DSP and beamforming for radar and TLC applications.
Marina Diomedi is Head of the Cerebrovascular Disease Center and Neurosonology Lab., Stroke Unit, University of Rome Tor Vergata. Clinical research activity on cerebrovascular diseases, particularly on ultrasound applications in acute stroke, stroke prevention and cerebral hemodynamics. 